\title{
SOFI-based 3D superresolution sectioning with a widefield microscope
}

\author{
Thomas Dertinger ${ }^{1,2^{*}}$, Jianmin $\mathrm{Xu}^{1}$, Omeed Foroutan Naini ${ }^{1}$, Robert Vogel ${ }^{1}$ and Shimon Weiss ${ }^{1,3,4^{*}}$
}

\begin{abstract}
Background: Fluorescence-based biological imaging has been revolutionized by the recent introduction of superresolution microscopy methods. 3D superresolution microscopy, however, remains a challenge as its implementation by existing superresolution methods is non-trivial.

Methods: Here we demonstrate a facile and straightforward 3D superresolution imaging and sectioning of the cytoskeletal network of a fixed cell using superresolution optical fluctuation imaging (SOFI) performed on a conventional lamp-based widefield microscope.
\end{abstract}

Results and Conclusion: SOFl's inherent sectioning capability effectively transforms a conventional widefield microscope into a superresolution 'confocal widefield' microscope.

\section{Background}

Superresolution (SR) imaging has revolutionized fluorescence biological imaging by providing resolution enhancement down to a few 10's of nanometers, allowing us to decipher morphology of small organelles and sub-cellular structures (S. Hell 2009; Huang et al. 2009; Schermelleh et al. 2010). Superresolution methods, however, generically provide enhanced resolution in two dimensions (2D) only (Betzig et al. 2006; Rittweger et al. 2009; Rust et al. 2006); additional resolution enhancement along the optical axis (i.e. SR in three dimensions, 3D) is a more challenging task and usually requires additional (and often significant) modifications to the optical set-up (Nagorni and Hell 2001; Pavani et al. 2009; Roman et al. 2008; Shtengel et al. 2009). Other technical challenges have to do with sample preparation protocols. For example, extra care is required to avoiding refractive index changes, since those could adversely affect the quality of the point spread function (PSF) (Huang et al. 2008; Klar 2000). The illumination/ excitation arm of the microscope often needs to be modified as well (Carlton 2008; Gustafsson et al. 1999; Juette et al. 2008). Furthermore, in single-molecule localization methods, the imaging depth that supports

\footnotetext{
* Correspondence: thomas.dertinger@gmail.com; sweiss@chem.ucla.edu 'Department of Chemistry and Biochemistry, University of California Los Angeles, Los Angeles, CA, USA

${ }^{2}$ SOFast GmbH, Dresdener Str 14, 10999 Berlin, Germany

Full list of author information is available at the end of the article
}

good 3D PSF fitting is very limited because of optical aberrations that rapidly degrade the PSF along the optical axis (z). In structured illumination 3D SR methods, such as 4Pi and ISO STED, refractive index changes are causing even more severe problems, because distorted wavefronts (of excitation and/or depletion beams) lead to loss of resolution and/or incomplete depletion. However, despite these challenges, 3D images with unprecedented clarity were already resolved far below $100 \mathrm{~nm}$ along the optical axis (Aquino et al. 2011). Recently we developed a novel approach to superresolution, which we termed Superresolution Optical Fluctuation Imaging (SOFI) (Dertinger et al. 2009; Dertinger et al. 2010a; Dertinger et al. 2010b). We were able to show that SOFI works on data acquired by a conventional lamp-based widefield microscope without any hardware modifications, achieving a factor of five in resolution enhancement using quantum dots (and a factor of four using conventional dyes (Dertinger et al. 2010b; Geissbuehler et al. 2011) while concomitantly eliminating background and out-of-focus light. Further, we showed that the achieved resolution enhancement is taking place along all three dimensions. Here we expand on the inherent $3 \mathrm{D}$ sectioning and the enhanced axial resolution attributes of SOFI by demonstrating straightforward 3D superresolution imaging of a whole cell using a widefield, lamp-based optical microscope. 


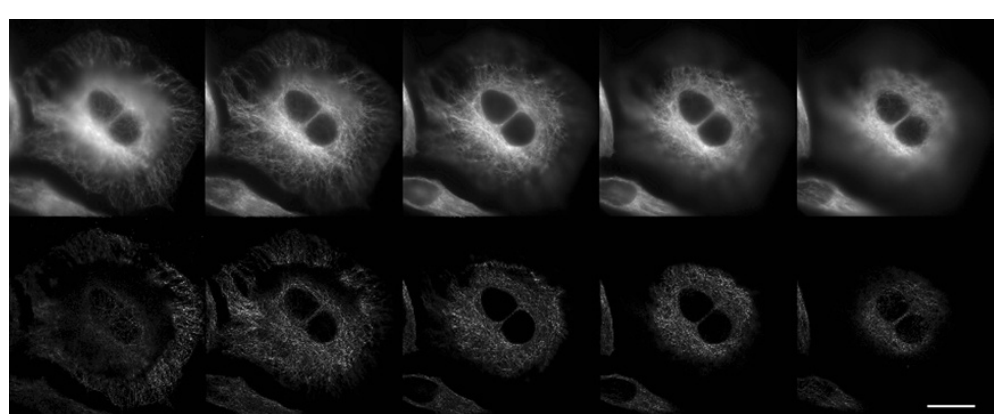

Figure 1 Lateral cross-sections. Subset of images recorded along the optical axis (in steps of 1.2um, starting with e lowest slice on the left side). Upper panels: Original widefield fluorescence images Lower panels: SOFI images. The optical sectioning capabilities are evident as well as the resolution gain along $x-y$. The SOFI images were processed using 2000 frames per z-position. Fluorescence images are representing the average of 2000 frames. Scalebar $20 \mu \mathrm{m}$.

\section{Methods}

The tubulin network of fixed HELA cells was immunostained with infrared emitting quantum dots (QD800, LifeTec, USA). A conventional LED-based (Aura light engine, Lumencor Inc., USA) widefield microscope (Nikon Eclipse Ti, Nikon Inc. USA) equipped with a piezo objective holder was used to successively acquire movies at different foci. 2000 frames were acquired per given height, with 32 height steps of $200 \mathrm{~nm}$ each. Excitation wavelength was $460-480 \mathrm{~nm}$. Data were recorded using a $600 \mathrm{~nm}$ long pass. The acquisition time per given height was 60s. Each movie (single height) was processed using the SOFI algorithm adjusted for time lag zero only using cross-cumulants (see (Dertinger et al. 2010a)). Data analysis was done with home-written Matlab routines. Imaris (Bitplane, USA) was used for 3D rendering.

\section{Results and discussion}

Figure 1 shows a sub-set of the original widefield fluorescence and corresponding SOFI images of the tubulin network of fixed HeLa cells immuno-stained with infrared emitting quantum dots (the fully 3D-rendered cell can be found in the Additional files 1 and 2, displaying at a x10 stretched optical axis). The sub-set represent a stack of different height images with height steps of $1.2 \mu \mathrm{m}$ (skipping 6 slices at a time, $0 \mu \mathrm{m}, 1.2 \mu \mathrm{m}, 2.4 \mu \mathrm{m}$ etc.) along the $\mathrm{z}$-axis. The sectioning, background suppression and resolution enhancement afforded by SOFI are apparent for the stack. To determine the resolution gain along the optical axis, we took an $\mathrm{x}-\mathrm{z}$ cross-section of the 3D stack and analyzed an intensity profile along the z-direction. Such an analysis indeed demonstrates an increased resolution along the optical axis, as shown in Figure 2. The resolution enhancement along the optical

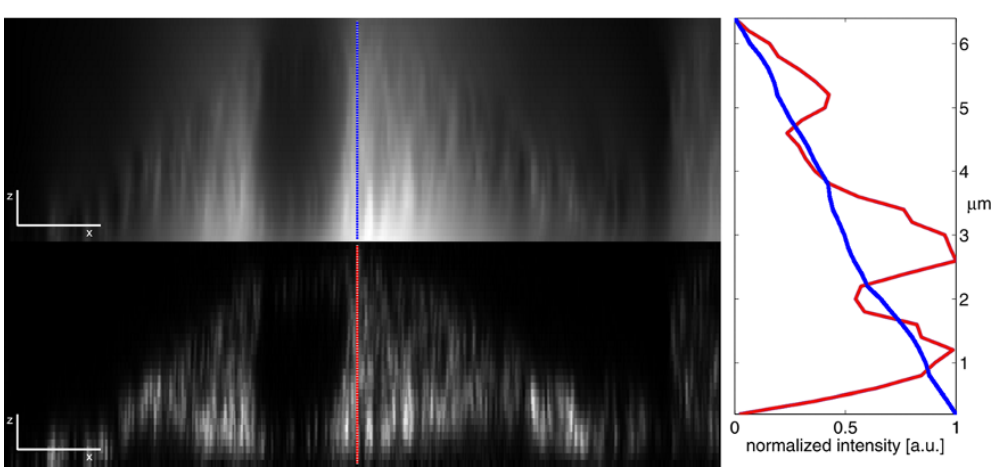

Figure 2 Vertical cross-sections. Vertical $(x-z)$ cross-sections of the three dimensional dataset. The scale for the $z$-axis is $x 10$ times that of the $x-$ axis. Upper panel: Original fluorescence image. Lower panel: SOFI image. Nearest neighbor smoothing was performed for the SOFI image in order to filter high frequency noise originated from long blinking intermittency of the quantum dots along the $z$ axis (the acquisition time of each slice is 60s). Blue and red lines indicate the same cross-sections for the original and SOFI images respectively. Right Panel: the corresponding crosssections for the original (red) and the SOFI (blue) images. The SOFI intensity profile clearly shows increased resolution along z. Scalebar: $1 \mu \mathrm{m}$ along $z$ and $20 \mu \mathrm{m}$ along $x$. 


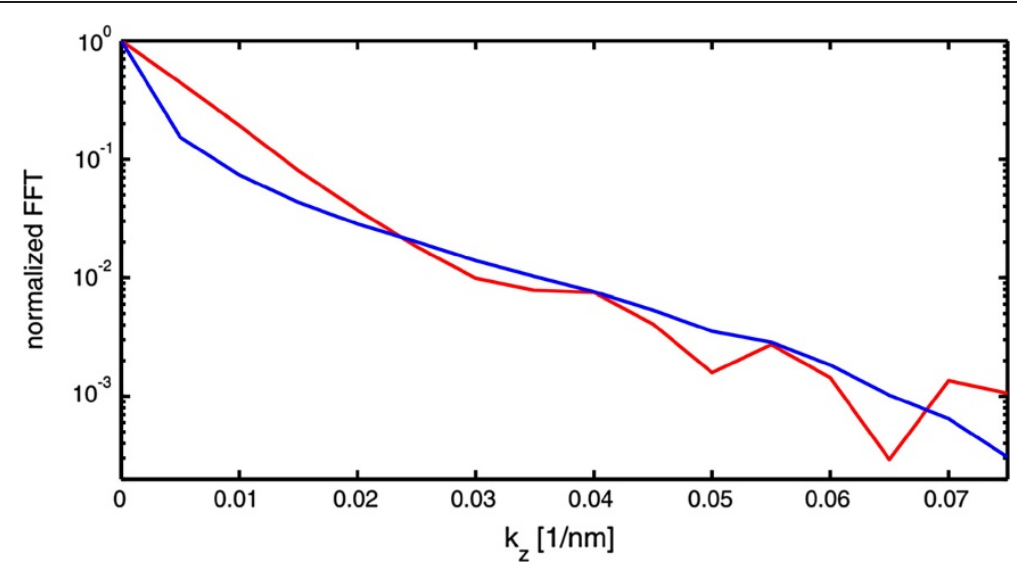

Figure 3 FFT-Spectrum. FFT spectra along the axis $k_{x}=k_{y}=0$ axis of the original fluorescence and SOFI data. Blue line: Fluorescence. Red line: SOFI. A clear cut-off frequency cannot be determined. Therefore the support of both these spectra cannot be estimated.

axis is however difficult to estimate, since the axial resolution of the original widefield data set is ill-defined (a diffraction limited axial resolution is well defined for a confocal microscope, but not for a widefiled microscope). We can however give an upper bound for the SOFI resolution along the $\mathrm{z}$-axis of $1.2 \mu \mathrm{m}$, as this is the distance between the resolved peaks in Figure 2.

We also evaluated the normalized FFT power spectrum of both (widefield and SOFI) data sets along the $\mathrm{k}_{\mathrm{x}}=\mathrm{k}_{\mathrm{y}}=0$ axis in order to examine if the support of the original FFT spectrum is indeed extended for the SOFI processed data set. An extended support is the manifestation of the achieved superresolution along the optical axis. The FFT spectra shown in Figure 3 indeed differ from each other, however a clear cut-off frequency cannot be deduced, leaving the determination of the resolution enhancement inconclusive (we note, however, that in a previous publication we were able demonstrate
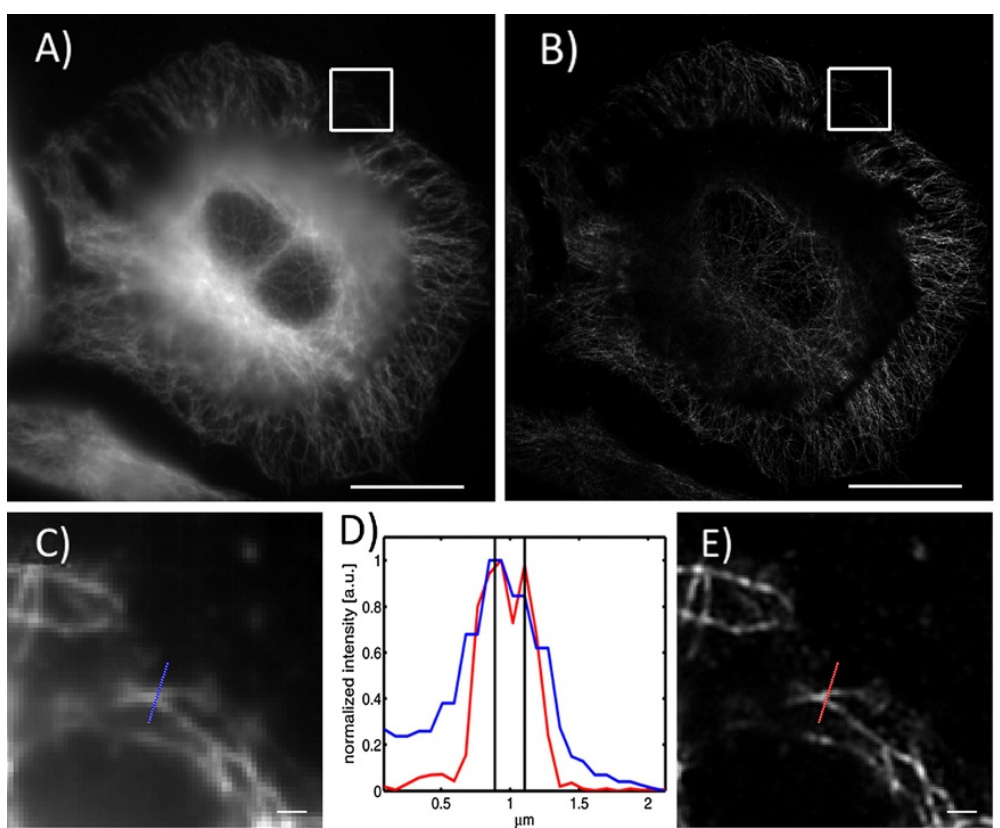

Figure $\mathbf{4}$ Lateral resolution gain. Estimating the resolution gain in the $\mathrm{x}$-y-plane. A) Fluorescence image. B) SOFI image. The boxed regions are displayed magnified and intensity-normalized in $\mathbf{C}$ ) and $\mathbf{E}$ ). Blue and red lines indicate where the cross-section was taken in order to evaluate the resolution gain. D): intensity profiles along the lines of $\mathbf{C}$ ) and $\mathbf{E}$ ). Blue line: Cross-section of the original fluorescence image. Red line: Cross-section of the SOFI image. As can be seen the blue line displays a dip indicating a higher resolution. The distance between the vertical black lines amounts to $215 \mathrm{~nm}$, which is $14 \%$ higher than the theoretically expected resolution of $188 \mathrm{~nm}$. Scalebar in A) and B) $20 \mu \mathrm{m}$; C) and E) $1 \mu \mathrm{m}$. 
the axial resolution enhancement by measuring the PSF of a single blinking QDs (Dertinger et al. 2009)). Since different slices were acquired at different times, the gain in axial resolution was somewhat compromised. Ideally, several z-slices should be acquired simultaneously in order to simultaneously capture blinking statistics of different voxels along $\mathrm{z}$.

To prove concurrent resolution enhancement in $\mathrm{x}, \mathrm{y}$ and $z$, we also estimated the resolution gain in the lateral direction ( $x$ - $y$-plane) by taking an intensity crosssection of a single image $\mathrm{z}$-slice. The result is shown in Figure 4. As expected, the resolution gain amounts to almost a factor of two. The theoretical resolution limit can be calculated using the Rayleigh criterion: $0.61 * 800 \mathrm{~nm} / 1.3=375 \mathrm{~nm}$. Therefore for the SOFI image we expected a resolution of no better than $188 \mathrm{~nm}$, which is the limit for a noise-free dataset. Since our dataset contains noise, the achievable resolution will be less than a factor of two. The achieved SOFI resolution was $215 \mathrm{~nm}$, slightly higher than expected, most likely due to non-perfect optics and noise.

\section{Conclusion}

We demonstrated SOFI-based 3D superresolution imaging of a whole cell using a conventional, unmodified, widefield microscope. These findings demonstrate the power and increased utility of the SOFI algorithm and could possibly allow extending these capabilities to deep tissue 3D superresolution imaging in the future. While QDs have many advantages, a drawback for SOFI analysis is their non-trivial power-law intermittency (blinking) behavior (Kuno et al. 2000). While for the second-order SOFI images the power-law blinking is not problematic, it has a stronger impact on higher order SOFI images (see discussion in (Dertinger et al. 2009)). A Poisson blinking process (as for triplet states of dyes and fluorescent proteins) does not suffer from this limitation and therefore is more amenable for higher order analysis.

Further axial resolution enhancement is expected if a multi-z-view acquisition is employed.

\section{Additional files}

Additional file 1: Shows the 3D rendered version of the original data set. The optical axis is displayed $\times 10$ stretched.

Additional file 2: Shows the 3D rendered version of the SOFI data set. The optical axis is displayed $\times 10$ stretched.

\section{Competing interests}

TD and SW are shareholders of SOFast GmbH Berlin, a company aiming for the commercial advancement and exploration of the SOFI technology. All other Authors declare that they do not have competing interests.

\section{Acknowledgements}

This work was supported by the National Institute of Health (NIH) grant\# 5R01EB000312 and grant\# 1R01GM086197 (to SW).

\section{Author details}

${ }^{1}$ Department of Chemistry and Biochemistry, University of California Los Angeles, Los Angeles, CA, USA. ${ }^{2}$ SOFast GmbH, Dresdener Str 14, 10999 Berlin, Germany. ${ }^{3}$ Department of Physiology University of California Los Angeles, UCLA, Los Angeles, USA. ${ }^{4}$ California NanoSystems Institute University of California Los Angeles, UCLA, Los Angeles, USA.

\section{Authors' contributions}

TD: designed experiments, evaluated the data, prepared the manuscript. JXU: prepared the QDs for immuno-staining. OF: performed measurements. RV: designed experiments and performed measurements. SW: designed experiments and prepared the manuscript. All authors approved. All authors read and approved the final manuscript.

Received: 24 November 2011 Accepted: 25 January 2012

Published: 25 April 2012

\section{References}

Aquino D, Schönle A, Geisler C, Middendorff CV, Wurm CA, Okamura Y, Lang T et al (2011) Two-color nanoscopy of three-dimensional volumes by 4Pi detection of stochastically switched fluorophores. Nat Methods 8(4):353-359. doi:10.1038/ nmeth.1583, Nature Publishing Group, a division of Macmillan Publishers Limited. All Rights Reserved

Betzig E, Patterson GH, Sougrat R, Lindwasser OW, Olenych S, Bonifacino JS, Davidson MW et al (2006) Imaging intracellular fluorescent proteins at nanometer resolution. Science (New York, NY) 313(5793):1642-1645. doi:10.1126/science. 1127344

Carlton PM (2008) Three-dimensional structured illumination microscopy and its application to chromosome structure. Chromosome research: an international journal on the molecular, supramolecular and evolutionary aspects of chromosome biology 16(3):351-365. doi:10.1007/s10577-008-1231-9

Dertinger T, Colyer R, lyer G, Weiss S, Enderlein J (2009) Fast, background-free, 3D super-resolution optical fluctuation imaging (SOFI). Proc Natl Acad Sci U S A 106(52):22287-22292. doi:10.1073/pnas.0907866106

Dertinger T, Colyer R, Vogel R, Enderlein J, Weiss S (2010a) Achieving increased resolution and more pixels with Superresolution Optical Fluctuation Imaging (SOFI). Opt Express 18(18):18875. doi:10.1364/OE.18.018875, OSA

Dertinger T, Heilemann M, Vogel R, Sauer M, Weiss S (2010b) Superresolution optical fluctuation imaging with organic dyes. Angew Chem Int Ed Engl 49 (49):9441-9443. doi:10.1002/anie.201004138

Geissbuehler S, Dellagiacoma C, Lasser T (2011) Comparison between SOFI and STORM. Biomedical Optics Express 2(3):408-420, OSA

Gustafsson MGL, Agard DA, Sedat JW (1999) I5M: 3D widefield light microscopy with better than $100 \mathrm{~nm}$ axial resolution. J Microsc 195(1):10-16. doi:10.1046/ j.1365-2818.1999.00576.x, Citeseer

Hell S (2009) Microscopy and its focal switch. Nat Methods 6(1):24-32. doi:10.1038/nmeth.1291

Huang B, Bates M, Zhuang X (2009) Super-resolution fluorescence microscopy. Annu Rev Biochem 78:993-1016. doi:10.1146/annurev. biochem.77.061906.092014, Annual Reviews

Huang B, Jones SA, Brandenburg B, Zhuang X (2008) Whole-cell 3D STORM reveals interactions between cellular structures with nanometer-scale resolution. Nat Methods 5(12):1047-1052. doi:10.1038/nmeth.1274, Nature Publishing Group

Juette MF, Gould TJ, Lessard MD, Mlodzianoski MJ, Nagpure BS, Bennett BT, Hess ST et al (2008) Three-dimensional sub-100 nm resolution fluorescence microscopy of thick samples. Nat Methods 5(6):527-529, New York: Nature Pub. Group, [2004]

Klar TA (2000) Fluorescence microscopy with diffraction resolution barrier broken by stimulated emission. Proc Natl Acad Sci 97(15):8206-8210. doi:10.1073/ pnas.97.15.8206

Kuno M, Fromm DP, Hamann HF, Gallagher A, Nesbitt DJ (2000) Nonexponential "blinking" kinetics of single CdSe quantum dots: A universal power law behavior. J Chem Phys 112(7):3117-3120. doi:10.1063/1.480896

Nagorni M, Hell SW (2001) Coherent use of opposing lenses for axial resolution increase in fluorescence microscopy. I. Comparative study of concepts. J Opt Soc Am A 18(1):36. doi:10.1364/JOSAA.18.000036, OSA 
Pavani SRP, Thompson MA, Biteen JS, Lord SJ, Liu N, Twieg RJ, Piestun R et al (2009) Three-dimensional, single-molecule fluorescence imaging beyond the diffraction limit by using a double-helix point spread function. Proc Natl Acad Sci U S A 106(9):2995-2999. doi:10.1073/pnas.0900245106

Rittweger E, Han KY, Irvine SE, Eggeling C, Hell S (2009) STED microscopy reveals crystal colour centres with nanometric resolution. Nature Photonics 3(3):144-147. doi:10.1038/nphoton.2009.2, Nature Publishing Group

Roman Schmidt, Alexander Egner, Stefan W, Hell SW (2008). isoSTED Microscopy - OSA Technical Digest (CD). Frontiers in Optics (p. FTuS2). Optical Society of America.

Rust MJ, Bates M, Zhuang X (2006) Sub-diffraction-limit imaging by stochastic optical reconstruction microscopy (STORM). Nat Methods 3(10):793-795. doi:10.1038/nmeth929

Schermelleh L, Heintzmann R, Leonhardt H (2010) A guide to super-resolution fluorescence microscopy. J Cell Biol 190(2):165-175. doi:10.1083/jcb.201002018

Shtengel G, Galbraith JA, Galbraith CG, Lippincott-Schwartz J, Gillette JM, Manley $\mathrm{S}$, Sougrat R et al (2009) Interferometric fluorescent super-resolution microscopy resolves 3D cellular ultrastructure. Proc Natl Acad Sci U S A 106 (9):3125-3130, National Acad Sciences

doi:10.1186/2192-2853-1-2

Cite this article as: Dertinger et al: SOFI-based 3D superresolution sectioning with a widefield microscope. Optical Nanoscopy 2012 1:2.

\section{Submit your manuscript to a SpringerOpen ${ }^{\circ}$} journal and benefit from:

- Convenient online submission

- Rigorous peer review

- Immediate publication on acceptance

- Open access: articles freely available online

- High visibility within the field

- Retaining the copyright to your article

Submit your next manuscript at $>$ springeropen.com 